\title{
Versatile Phase Transfer Method for the Efficient Surface Functionalization of Gold Nanoparticles: Towards Controlled Nanoparticle Dispersion in a Polymer Matrix
}

\author{
Katarzyna Ranoszek-Soliwoda, ${ }^{1}$ Maria Girleanu, ${ }^{2,3}$ \\ Beata Tkacz-Szczęsna, ${ }^{1}$ Marcin Rosowski, ${ }^{1}$ Grzegorz Celichowski, ${ }^{1}$ \\ Martin Brinkmann, ${ }^{2}$ Ovidiu Ersen, ${ }^{3}$ and Jaroslaw Grobelny ${ }^{1}$ \\ ${ }^{1}$ Department of Materials Technology and Chemistry, Faculty of Chemistry, University of Lodz, Pomorska 163, 90-236 Lodz, Poland \\ ${ }^{2}$ Institut Charles Sadron, UPR-22 CNRS, 23 rue du Loess, BP 84047, 67034 Strasbourg Cedex 2, France \\ ${ }^{3}$ Institut de Physique et Chimie des Matériaux de Strasbourg, UMR 7504 CNRS-UdS, 23 rue du Loess BP 43, \\ 67034 Strasbourg Cedex 2, France \\ Correspondence should be addressed to Jaroslaw Grobelny; jgrobel@uni.lodz.pl
}

Received 9 March 2016; Revised 13 May 2016; Accepted 22 May 2016

Academic Editor: Ilaria Fratoddi

Copyright ( 2016 Katarzyna Ranoszek-Soliwoda et al. This is an open access article distributed under the Creative Commons Attribution License, which permits unrestricted use, distribution, and reproduction in any medium, provided the original work is properly cited.

\begin{abstract}
In electronic devices based on hybrid materials such as nonvolatile memory elements (NVMEs), it is essential to control precisely the dispersion of metallic nanoparticles (NPs) in an insulating polymer matrix such as polystyrene in order to control the functionality of the device. In this work the incorporation of AuNPs in polystyrene films is controlled by tuning the surface functionalization of the metallic nanoparticles via ligand exchange. Two ligands with different structures were used for functionalization: 1-decanethiol and thiol-terminated polystyrene. This paper presents a versatile method for the modification of gold nanoparticles (AuNPs) with thiol-terminated polystyrene ligands via phase transfer process. An organic colloid of AuNPs ( $5 \pm 1 \mathrm{~nm}$ diameter) is obtained by the phase transfer process (from water to toluene) that allows exchanging the ligand adsorbed on AuNPs surface (hydrophilic citrate/tannic acid to hydrophobic thiols). The stability, size distribution, and precise location of modified AuNPs in the polymer matrix are obtained from UV-Vis spectroscopy, dynamic light scattering (DLS), and electron tomography. TEM tomographic 3D imaging demonstrates that the modification of AuNPs with thiol-terminated polystyrene results in homogeneous particle distribution in the polystyrene matrix compared to 1-decanethiol modified AuNPs for which a vertical phase separation with a homogeneous layer of AuNPs located at the bottom of the polymer matrix was observed.
\end{abstract}

\section{Introduction}

Nowadays, almost every branch of science and industry exploits the unique properties of nanoparticles (NPs) that can be tuned by adjusting precisely their nanometric size and shape. In biology NPs are used as tools for drug delivery $[1,2]$, imaging [1], and sensing [1,3-5]. In catalysis, NPs help to enhance the selectivity and efficiency of heterogeneous catalysis $[6,7]$, whereas in tribology material wear resistance can be adjusted $[8,9]$. In some cases, the incorporation of polydisperse NPs allows obtaining a new material with expected properties, whereas the use of monodisperse NPs with defined and very well controlled shape and homogeneity in size is more stringent in electronics applications, especially in case of nonvolatile memory elements (NVMEs). Moreover, the use of NPs in electronic applications requires the adjustment of NPs composition (the core material of the NPs, the substances present on NPs surface as well as substances present in the colloid solution, etc.) to the manufacturing technology of hybrid materials.

There are two main roads to incorporate chemically synthesized NPs into memory element: the first one is the deposition of NPs from aqueous colloids onto memory element without a direct contact of solvent (water) with the 
element surface (e.g., via electrospray deposition technique [10]). The second method is based on the spin-coating or ink-jet printing of polymer inks containing NPs dispersed in nonpolar solvent (e.g., toluene, hexane, dichlorobenzene, and anisole) which will preserve the memory element structure. In both cases, the use of monodisperse NPs with well defined size and very narrow size distribution is mandatory to control precisely the incorporation of metallic NPs (mNPs) in an organic matrix (polymer or molecular semiconductor or insulator) and hence the functionality of the hybrid layers. Moreover, the use of monodisperse mNPs allows the modelling of the system and thus comparison and understanding of the phenomenon responsible for the properties and functionality of such systems. Existing explanation of the role of nanoparticles in the process of resistive memory switching mechanisms is based on two different phenomena. One is the change of electrical conductance between two electrodes that depends on the amount of charge stored or trapped, on the metallic nanoparticles. Depending on the voltage applied to electrodes, nanoparticles may be charged or discharged and, as a consequence, provide two different electrical states essential for the functioning of the memory [11]. The second phenomenon is the local change of electrical conductance based on filament formation. The simplest form of filament is a metallic bridge connecting the two electrodes. Filament formation or rupture provides two electrical states of low or high conductance [12]. In both mentioned cases mNPs nanoparticles play an important role in memory functionality.

To incorporate the mNP within the polymer matrix in order to produce polymer inks, two main approaches were followed so far: the first one is the in situ synthesis of NPs from metal precursor directly inside the polymer matrix $[13,14]$ and the second one is the ex situ method where tailored and presynthesized NPs are incorporated into the polymer matrix $[15,16]$. The in situ approach has several limitations mainly related to the difficulties in controlling the NPs uniformity in size. In turn, the ex situ approach is more straightforward as it makes use of presynthesized NPs with desired uniformity in size and shape and hence is much more suitable for electronic applications. Furthermore, the location of particles in polymer matrix can be easily and precisely controlled by tailoring the surface properties of NPs. The distribution of presynthesized NPs in a polymer matrix can be controlled by surface modification using different compounds, for example, dendrimers [17], peptides [18], and polymers $[19,20]$, which can be attached to the NPs via grafting-from, grafting-to, ligand exchange, or templating methods. In the grafting-from method, ligands (mainly polymers) are usually grown directly from the NP surfaces via living free radical polymerization [21, 22]. Alternatively, presynthesized end-functionalized polymers can be directly grafted to the NP surface [23, 24]. Regarding templating methods, polymers (e.g., di- or triblock copolymer micelles and star polymers) are used as templates for NPs [25-27].

Finally, the ligand exchange method involves the exchange of adsorbed ligands on NPs [28, 29]. The main requirement and limitation of this method are that the final ligands should have a stronger affinity to the NPs surface compared to the initial ligands. Notwithstanding, this method is fast and versatile and allows using highly monodispersed NP colloids prepared according to wellknown and optimized procedures.

Monodisperse NPs can be obtained in both polar (e.g., via chemical reduction method [30-32]) and nonpolar solvents (e.g., using the Brust-Schiffrin method [33, 34]). However, the syntheses of mNPs in nonpolar solvents have some limitations, which are mainly related to the limited NPs size (usually below $10 \mathrm{~nm}$ ) $[35,36]$. Furthermore, mNPs in nonpolar solvents are usually synthesized in the presence of thiols or amines, which are very difficult to exchange with other ligands. Synthesis of mNPs in aqueous solvents is much more versatile and makes it possible to synthesize NPs with a wide range of sizes and composition depending on the synthesis method and conditions of the process [32].

For the incorporation of mNPs via electrospray deposition, aqueous NPs colloids synthesized via well-known and optimized procedures (e.g., chemical reduction method [32]) can be used. Instead, in the case of polymer inks, mNPs must be soluble in common organic solvents such as toluene. The organic colloids of mNPs are obtained via modification of aqueous synthesized NPs with hydrophobic ligands (e.g., thiols and amines) using a phase transfer process from water to nonpolar solvents [30, 31]. Organic colloids can then be used for the preparation of polymer inks suitable for spincoating or ink-jet printing to fabricate nonvolatile memory elements.

The modification of mNPs is important not only to obtain a stable polymer ink but also to ensure homogeneous distribution of mNPs in the polymer matrix after the ink deposition onto memory element and solvent evaporation. The problem with inhomogeneous distribution of mNPs in polymer layer was presented by Corbierre et al. [23, 37, 38]. The authors showed that the incorporation of NPs in a polymer matrix is thermodynamically more favorable when the NPs are functionalized with a ligand that is chemically similar to the polymer matrix [37]. This method implies a rather complex synthesis of thiol-terminated polystyrene ligand according to Stouffer and McCarthy [39]. Moreover, this NPs' preparation necessitates a purification step of the colloid to eliminate by-products of the synthesis by evaporation of the colloid and washing the precipitate with an appropriate solvent. This in turn may result in NPs aggregation. Another difficulty of this protocol is that the synthesis of other types of NPs (e.g., silver and platinum) modified with thiolterminated polystyrene will require the specific optimization of the synthesis conditions.

As mentioned previously, the shape, the size distribution, and the location of mNPs are important parameters for the optimization of the expected properties of hybrid systems [11].

Besides UV-Vis spectroscopy or dynamic light scattering (DLS) which are applied for evaluating the size and the stability of metallic colloids in solution, the characterization of hybrid films requires other methods such as transmission electron microscopy (TEM) to characterize the bulk distribution of NPs in the polymer matrix [22]. Conventional 2D TEM analysis provides proper information on size and 
density of mNPs on a given surface. However, for a precise location of NPs within the bulk volume of a thin film, electron tomography is necessary. TEM tomography is able to reconstruct the $3 \mathrm{D}$ morphology of complex polymer-based systems such as gyroid phases of block copolymers or the NPs location in hybrid systems made of mNPs dispersed in a copolymer matrix [40-44]. Other techniques such as superresolution fluorescence microscopy [45] can also be used for determination of the spatial arrangement of organic and inorganic phases in advanced materials (e.g., biological organic-inorganic hybrid materials).

This paper presents a versatile method for AuNPs surface functionalization with thiol-terminated polystyrene using a simple phase transfer method in order to prepare AuNPspolystyrene inks of interest for the preparation of nonvolatile hybrid memories. This method is based on the use of an aqueous colloid of mNPs and its modification with commercially available thiol-terminated polystyrene $\left(M_{w}=11.000\right)$ by phase transfer method from water to toluene. The presented method is flexible and is based on the modification of mNPs prepared in aqueous solvent according to well-known and optimized procedure (i.e., chemical reduction method). Regarding the method used hereafter, the synthesis of mNPs is carried out by the reduction of metal salt using: sodium citrate and tannic acid which can be next easily replaced by other ligands that have greater affinity to the surface of the NPs (e.g., hydrophobic ligands like thiols) via phase transfer process from water to toluene [30,31]. This allows obtaining a stable organic colloid that does not require purification steps, as all by-products remain in water after a phase transfer process. The size and stability of modified AuNPs were monitored with DLS and UV-Vis spectroscopy. Moreover, the density and the location of mNPs within the bulk volume of polymer matrix were investigated by conventional TEM and electron tomography.

\section{Materials and Methods}

2.1. Materials. Gold(III) chloride hydrate (Sigma-Aldrich, $\geq 49 \%$ ), tannic acid (Fluka), sodium citrate (Sigma-Aldrich, $\geq 99.0 \%$ ), 1-decanethiol (Sigma-Aldrich, 96\%), thiolterminated polystyrene (Sigma-Aldrich, $M_{n}=11.000$ ), and polystyrene (Sigma-Aldrich, analytical standard for GPC, $\left.M_{n}=2.500\right)$ were used as received. Toluene and acetone used for the phase transfer process were distilled before use. For all of the preparation of aqueous colloids deionized water obtained from Deionizer Millipore Simplicity UV system was used. The specific resistivity of water was $18.2 \mathrm{M} \Omega \cdot \mathrm{cm}$.

2.2. AuNPs Synthesis. AuNPs (5 nm diameter and concentration $100 \mathrm{ppm}$ ) were synthesized in water via chemical reduction following our previous work [31]. Aqueous solution of chloroauric acid (93.80 g, of $1.84 \cdot 10^{-2} \mathrm{wt} \%$ ) was boiled, at which point a mixture of sodium citrate $(4.48 \mathrm{~g}, 0,877 \mathrm{wt} \%)$ and tannic acid (1.73 g, $1 \mathrm{wt} \%)$ was added. Following the addition of the mixture, the solution colour changed from yellow to dark red. The suspension was stirred for additional $15 \mathrm{~min}$ and cooled down to room temperature. AuNPs colloids were used in the next steps for the preparation of polymer inks.
2.3. AuNPs Functionalization. AuNPs were modified with 1-decanethiol and thiol-terminated polystyrene. The ligand exchange was realized by using a phase transfer process from water to toluene. The samples will be referred to as $\mathrm{Au}$-Th for AuNPs modified with 1-decanethiol ligand (-Th) and Au-PS for the AuNPs modified with polystyrene thiolterminated ligand (-PS). For the phase transfer process each of the modifiers was prepared as a $0.01 \mathrm{wt} \%$. toluene solution. The modifier amount corresponds to 5 modifier molecules per $\mathrm{nm}^{2}$ of NP surface in both cases. Briefly, to the aqueous AuNPs colloid acetone and toluene with one of the modifier compounds were added. Acetone was used to reduce the surface tension between the water and toluene phases [46] and to increase the miscibility of aqueous and organic phases. The weight ratio of aqueous colloid to acetone and toluene was: $2: 1: 1$, respectively. The solutions undergo vigorous shaking for $5 \mathrm{~min}$ in the case of 1-decanethiol and $30 \mathrm{~min}$ for thiol-terminated polystyrene for ligand exchange and followed by a phase separation for $1 \mathrm{~h}$. The mobility of small molecules of -Th ligand is much higher compared to the large -PS ligand. Hence, the exchange of citrate/tannic acid ligand to -Th ligand occurs much faster compared to -PS ligand. Organic colloids of Au-Th and Au-PS were further used for the preparation of polymer inks.

2.4. Polymer Inks Preparation. Polymer inks with NPs were prepared based on the organic colloids of Au-Th and AuPS. Phase transfer process of AuNPs was realized with acetone to reduce the surface tension between the water and organic phase and to increase phase's miscibility and hence facilitate the exchange of ligand molecules on AuNPs surface. However, acetone is a polar solvent and even small amounts of this solvent in polymer ink used for preparation of hybrid memory devices may affect its proper functionality. Hence, before the inks preparation acetone was removed from the organic colloid by evaporation process. The organic colloid was heated to $60^{\circ} \mathrm{C}$ and acetone was removed by evaporation (acetone $T_{\mathrm{bp}}=57^{\circ} \mathrm{C}$ ) and consequently toluene colloids of $\mathrm{Au}-$ Th and Au-PS were obtained. Two toluene colloids of Au-Th and Au-PS with NPs weight concentration of gold of about $200 \mathrm{ppm}$ and $400 \mathrm{ppm}$ were used to prepare polymer inks.

Colloid $400 \mathrm{ppm}$ was prepared based on the colloid 200 ppm by evaporation of toluene. Polystyrene inks PS@AuTh (PS-polystyrene and thiol functionalized gold nanoparticles) and PS@Au-PS (PS-polystyrene and thio-polystyrene functionalized gold nanoparticles) were prepared by incorporation of polystyrene $\left(M_{n}=2.500\right)$ into the toluene colloids, in case of both NPs concentrations, $200 \mathrm{ppm}$ and $400 \mathrm{ppm}$, respectively. The weight ratio of polystyrene to toluene colloid was $3.5: 1$, respectively. Any further purification steps were not carried out.

2.5. PS@Au Film Preparation. To probe the effect of the AuNPs surface functionalization on the dispersion in the polymer matrix, hybrid films were prepared by spin-coating (2000 rpm for $180 \mathrm{~s}$ ) the AuNPs/polystyrene inks on ITO substrates. ITO substrates were cleaned by sequential sonication in acetone, ethanol, an aqueous solution of Hellmanex (Hellma, Germany), and distilled water (3x). Finally they 
were dried in a flow of nitrogen. To reduce electron beam damage and charging effects during TEM analysis, the films were coated with a thin amorphous carbon layer $(2-5 \mathrm{~nm}$ thick) using an Auto 306 evaporator. The $100-120 \mathrm{~nm}$ thick films were removed from ITO by floating on a $1 \mathrm{wt} \%$ solution of HF and recovered on TEM copper grids. The hybrid films did not undergo any annealing or staining treatment. The scheme of the preparation procedure of polymer films with functionalized AuNPs is shown in Supplementary Material (see Supplementary Material available online at http://dx.doi.org/10.1155/2016/9058323).

\subsection{Methods}

2.6.1. UV-Vis Spectroscopy. The UV-Vis spectra were recorded with a spectrophotometer USB2000 + detector (miniature fiber optic spectrometer), Ocean Optics, HL-2000 (tungsten halogen light sources) using $1 \mathrm{~cm}$ quartz cuvette.

2.6.2. Dynamic Light Scattering (DLS). The DLS of AuNPs in water and $\mathrm{Au}$-Th and $\mathrm{Au}$-PS in toluene were carried out with a DLS Nano ZS Zetasizer system (Malvern Instruments) with a He-Ne laser $(633 \mathrm{~nm})$ as the light source. The measurements were performed with a scattering angle of $173^{\circ}$ (a measurement temperature of $25^{\circ} \mathrm{C}$; aqueous colloids, medium viscosity $0.887 \mathrm{mPa} \cdot \mathrm{s}$, and material refractive index 1.330 ; toluene colloids, medium viscosity $0.556 \mathrm{mPa} \cdot \mathrm{s}$, dispersant refractive index 1.496, and material refractive index 1.330).

2.6.3. TEM. The experimental data for conventional TEM analysis and for electron tomography were acquired with a JEOL $2100 \mathrm{~F}$ transmission electron microscope equipped with a field emission gun operating at $200 \mathrm{kV}$, a spherical aberration probe corrector, and a GATAN Tridiem energy filter. Acquisitions of the tilt images series were performed using a high tilt sample holder, under angles spanning from +65 to -65 degrees, with projections taken every $2^{\circ}$ according to Saxton scheme. The irradiation damage was limited by using low electron doses. The acquisition of the 84 TEM tilt images series was achieved with a cooled CCD detector $(2048 \times 2048$ pixels with a pixel size of $0.2 \mathrm{~nm})$. The images were first roughly aligned using a cross-correlation algorithm. A refinement of this initial alignment and a precise determination of the tilt axis direction were then obtained using the IMOD software where the centers of several Au nanoparticles from the analyzed group were used as fiducial markers [47]. The volume reconstructions have been computed using an iterative approach consisting of a simultaneous algebraic reconstruction technique implemented using the TOMO3D software [48], the number of iterations not exceeding 40. Visualization and quantitative analysis of the final volumes were carried out using ImageJ software.

\section{Results and Discussion}

3.1. Nanoparticles Surface Modification. The modification of AuNPs with the size of about $5 \pm 1 \mathrm{~nm}$ was performed via a phase transfer of NPs from water to toluene with the respective ligand: 1-decanethiol $(\mathrm{Au}-\mathrm{Th})$ or thiol terminated



FigUre 1: UV-Vis spectra of AuNPs aqueous colloid $\left(\lambda_{\max }=521 \mathrm{~nm}\right)$, AuNPs modified with 1-decanethiol (Au-Th) $\left(\lambda_{\max }=517 \mathrm{~nm}\right)$, AuNPs modified with thiol-terminated polystyrene $(\mathrm{Au}-\mathrm{PS})\left(\lambda_{\max }=\right.$ $531 \mathrm{~nm}$ ), and water phases after phase transfer process.

polystyrene (Au-PS). The effects of NPs modifications were easily visible as a result of the NP transfer from water to toluene with the corresponding coloration/discoloration of the toluene and the water phases, respectively. The phase transfer of AuNPs is a result of the ligand exchange on the AuNPs surface from hydrophilic citrate/tannic acid that stabilize NPs in polar solvent (water) to the hydrophobic thiols that stabilize colloidally NPs in nonpolar solvent (toluene). The effectiveness of the modification process was monitored with UV-vis spectroscopy (Figure 1).

The absorption band maxima for modified NPs were recorded in the region characteristic for AuNPs, at $517 \mathrm{~nm}$ and $531 \mathrm{~nm}$ for $\mathrm{Au}$-Th and Au-PS, respectively. No characteristic band from AuNPs was observed in the water phases after the phase transfer process. These results demonstrate the efficiency of the phase transfer process and prove the controlled AuNPs modification with hydrophobic ligands, -Th and -PS, respectively.

The colloidal state of AuNPs after the modification process with -Th and -PS ligands was monitored by DLS and compared with the results obtained for aqueous colloid (Figure 2).

The hydrodynamic size of AuNPs remains almost unchanged after the modification with 1-decanethiol $(8 \pm$ $2 \mathrm{~nm})$ compared to the initial aqueous colloid $(9 \pm 2 \mathrm{~nm})$ (Figures 2(a) and 2(b)). The modification of AuNPs with thiol-terminated polystyrene ligand results in objects with the hydrodynamic size of about $33 \pm 9 \mathrm{~nm}$ (Figure 2(c)). The thiol-terminated polystyrene ligand $\left(M_{w}=11.000 \mathrm{~g} / \mathrm{mol}\right)$ is much bigger compared with aliphatic 1-decanethiol or citrate/tannic acid molecules. Hence, the hydrodynamic size of AuNPs modified with thiol-terminated polystyrene ligand (Au-PS) is bigger than the hydrodynamic size of $\mathrm{Au}-\mathrm{Th}$ 


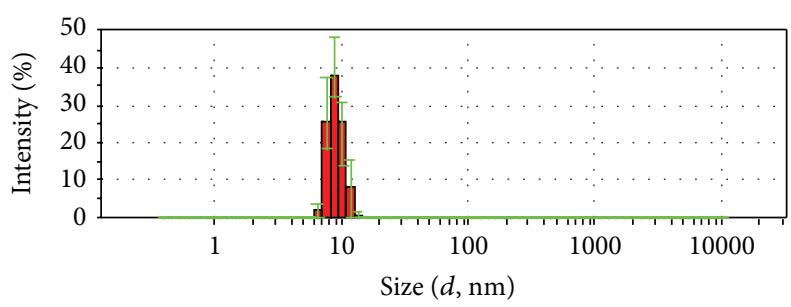

(a)



(b)

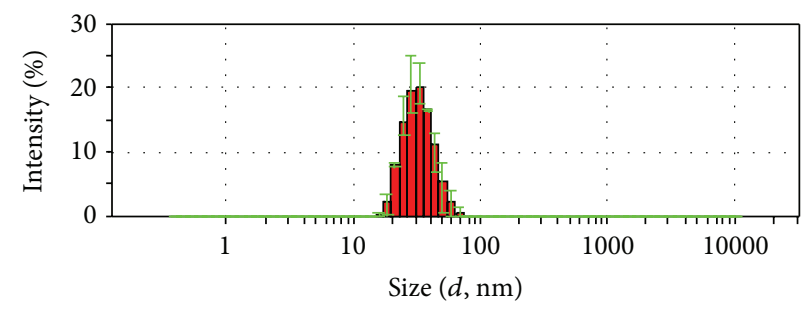

(c)

Figure 2: DLS size distribution histograms of (a) citrate/tannic acid stabilized AuNPs (aqueous colloid); (b) Au-Th (toluene colloid after a phase transfer of AuNPs with 1-decanethiol); (c) Au-PS (toluene colloid after a phase transfer of AuNPs with thiol-terminated polystyrene).

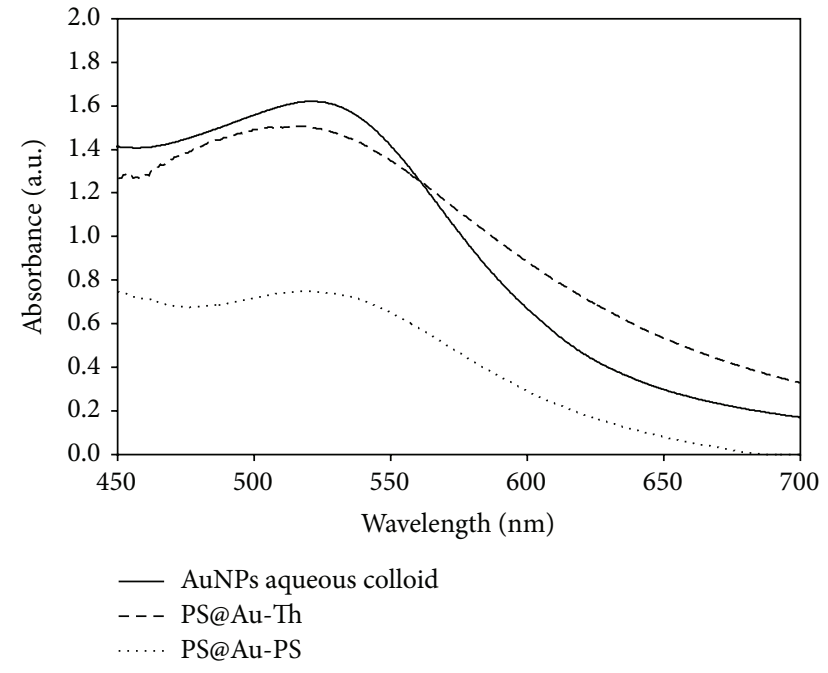

Figure 3: UV-Vis spectra of AuNPs aqueous colloid $\left(\lambda_{\max }=\right.$ $521 \mathrm{~nm}$ ) and polystyrene inks: PS@Au-Th (AuNPs modified with 1-decanethiol in polystyrene) $\left(\lambda_{\max }=531 \mathrm{~nm}\right)$; PS@Au-PS (AuNPs modified with polystyrene thiol terminated $)\left(\lambda_{\max }=531 \mathrm{~nm}\right)$.

or aqueous AuNPs colloid modified with 1-decanethiol or citric/tannic acid ligand, respectively.

3.2. Nanoparticles in Polymer Inks. The stability of Au-Th and Au-PS in polystyrene inks was monitored with UVVis spectroscopy (Figure 3 ). The absorption band maxima recorded in the region characteristic for AuNPs smaller than $30 \mathrm{~nm}$ [49] at $531 \mathrm{~nm}$ for both PS@Au-Th and PS@Au-PS prove the colloidal stability of NPs in polystyrene inks. Small changes in the position of the absorption band maxima compared to the aqueous colloid result from the changes in the refractive index of the surrounding medium (water and toluene) as well as NPs shell (citrate/tannic acid mixture and thiols) which have an impact on the local surface plasmon resonance (LSPR) of NPs.

3.3. Nanoparticles in Polymer Films. From a general point of view, the colloid solutions of AuNPs have to be processed under film form for electronic applications. In order to observe the influence of -Th or -PS functionalization of AuNPs on their dispersion within the polystyrene matrix, a first approach by using conventional TEM analyses was realized. The images showed in Figure 4 highlight a clear difference between both functionalizations.

In the case of -Th ligand a homogenous distribution of individual AuNPs is observed at $200 \mathrm{ppm}$ concentration and at 400 ppm concentration, as shown in Figures 4(a) and 4(c), respectively. For a higher concentration, the AuNPs seem to form single agglomerates of NPs but without metallic connections between individual particles (Figure 4(c)). The dispersion of individual AuNPs is about 5300 particles $/ \mu \mathrm{m}^{2}$ for $200 \mathrm{ppm}$ concentration and 9300 particles $/ \mu \mathrm{m}^{2}$ for $400 \mathrm{ppm}$ concentration, respectively. In the case of -PS ligands, the TEM analysis shows a homogenous dispersion of individual AuNPs for both concentrations and the presence of several AuNPs agglomerates (without metallic connections between individual NPs) (Figures 4(b) and 4(d)). The density of individual AuNPs $/ \mu \mathrm{m}^{2}$ for this functionalization is 625 particles $/ \mu \mathrm{m}^{2}$ for $200 \mathrm{ppm}$ concentration and 1000 particles $/ \mu \mathrm{m}^{2}$ for $400 \mathrm{ppm}$ concentration, respectively.

It is interesting to notice that the AuNPs agglomerates in the PS@Au-Th 400 ppm sample show regular hexagonal 2D packing suggesting 2D-type agglomerates. This observation opens the important question regarding the precise location of such $2 \mathrm{D}$-like aggregates in the hybrid layers. The precise 3D distribution of individual AuNPs and agglomerates on the surface or within the polystyrene layer for both types of ligands is an important parameter for understanding the 




(a)

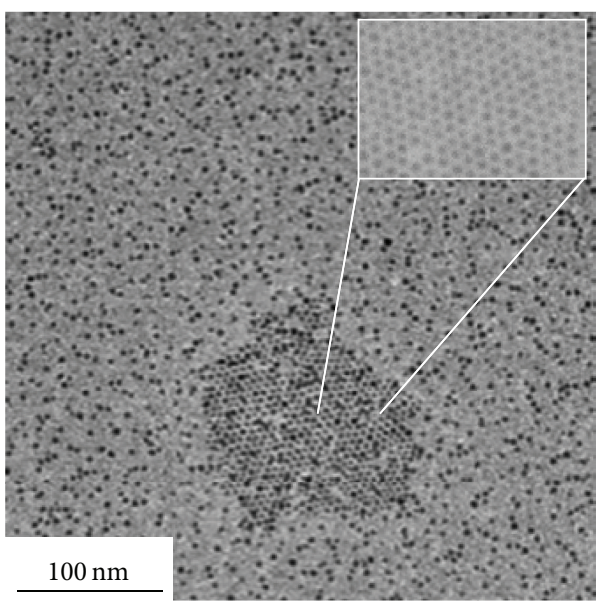

(c)

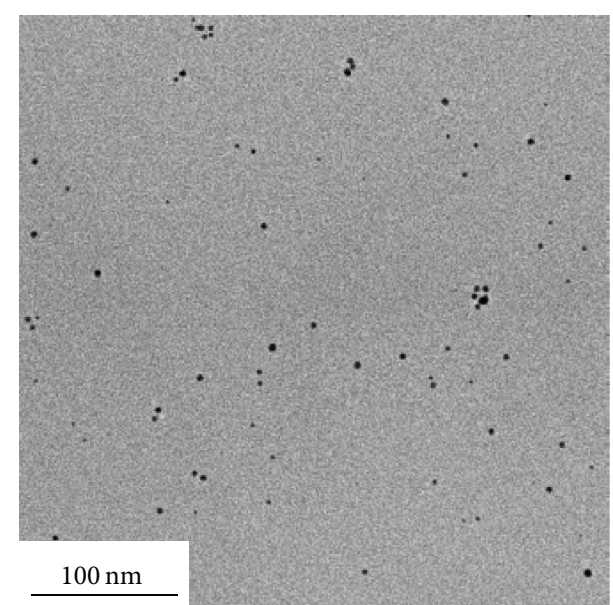

(b)

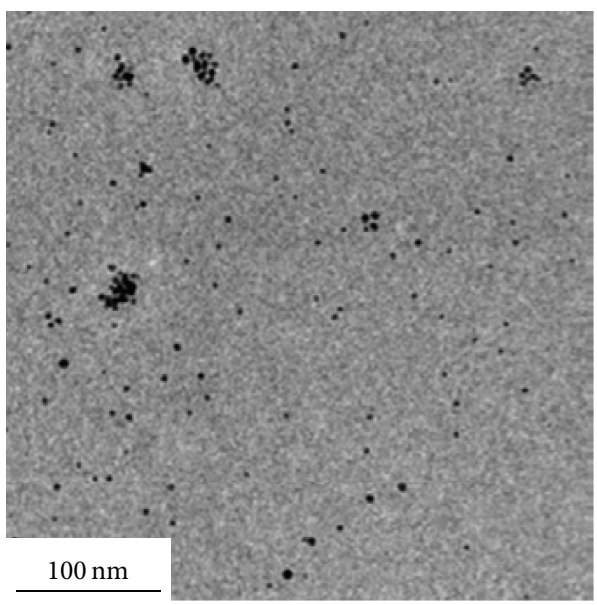

(d)

FIGURE 4: Bright field TEM images of PS@AuNPs for different functionalized ligands and different concentration in gold nanoparticles: (a) PS@Au-Th 200 ppm; (b) PS@Au-PS 200 ppm; (c) PS@Au-Th 400 ppm; (d) PS@Au-PS 400 ppm.

behaviour of such hybrid systems used in NVMEs, since such agglomerates might influence their switching mechanism. To obtain this information TEM tomography was used. The results of tomographic analysis for PS@Au samples with a concentration of 200 ppm in AuNPs are presented in Figure 5.

Representative areas of the PS@Au-Th and PS@Au-PS samples used for tomographic analysis are presented in Figures 5(a) and 5(b). In all samples, TEM tomography shows the coexistence of individually dispersed AuNPs and AuNPs agglomerates without metallic connections between NPs. However, as seen in Figures 5(c) and 5(d), the tomographic reconstruction reveals unambiguously a different location of the AuNPs within the bulk of the PS matrix depending on the surface ligands on the AuNPs. For the AuNPs functionalized with -Th ligands, a vertical phase separation is observed: a homogeneous layer of AuNPs is localized at the bottom of the polystyrene film (interface between ITO and PS film). As seen in the 2D TEM images, tomography confirms the formation of $2 \mathrm{D}$ agglomerates of AuNPs at the upper surface of the film (Figure 5(c)). The hexagonal packing of the AuNPs and the $2 \mathrm{D}$ character of these agglomerates indicates that they could be formed at the surface of the liquid film during the spincoating process. In any case, the segregation of all AuNPs to the film interfaces underlines the incompatibility of mixing between thiol-functionalized AuNPs and the polystyrene matrix.

In contrast, the use of the -PS ligand changes radically the distribution of the AuNPs within the PS matrix. In that case, the sum of cross-sectional images in the $x z$ plane reveals a uniform distribution of individual AuNPs and 3D aggregates within the bulk of the PS layer (Figure 5(d)). Contrary to the case of -Th ligands, no 2D agglomerates are formed on the surface of the hybrid layers but only $3 \mathrm{D}$ agglomerates that are embedded in the PS matrix. The fact that -PS ligand AuNPs form 3D ligands is possibly related to the entanglement of the PS chains belonging to adjacent AuNPs in these agglomerates. Such entanglements would favor the clustering of the AuNPs into 3D aggregates.

In addition to different AuNPs ligands functionalization, the increase in the AuNPs concentration is another parameter that can influence their distribution in the PS matrix. The results concerning the films prepared from PS@Au with 


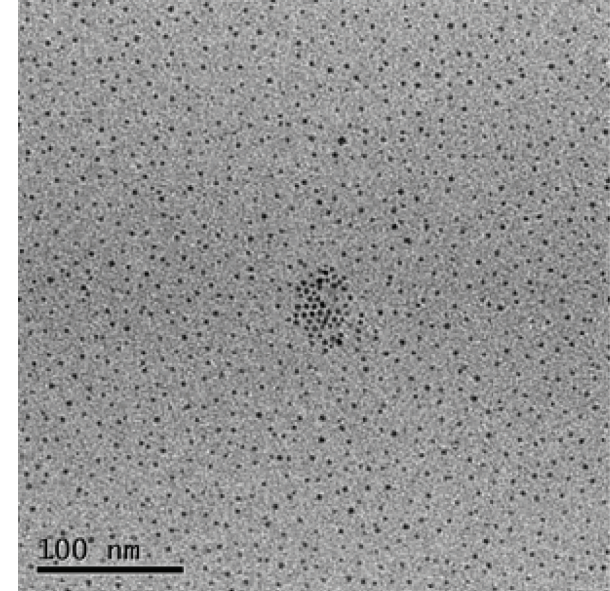

(a)



(c)

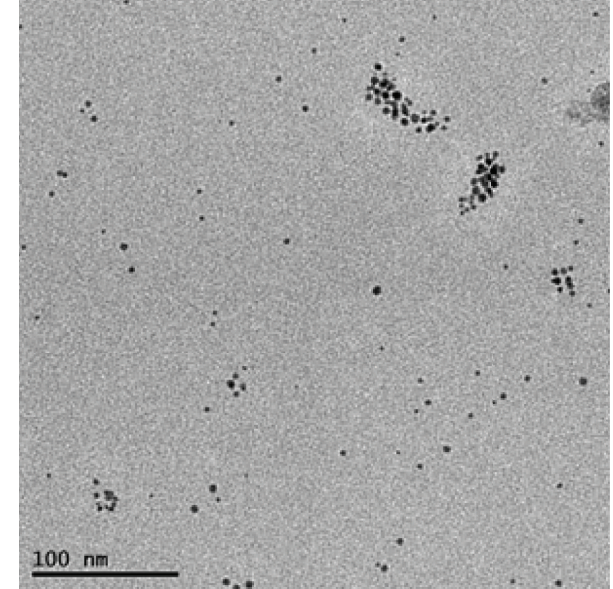

(b)

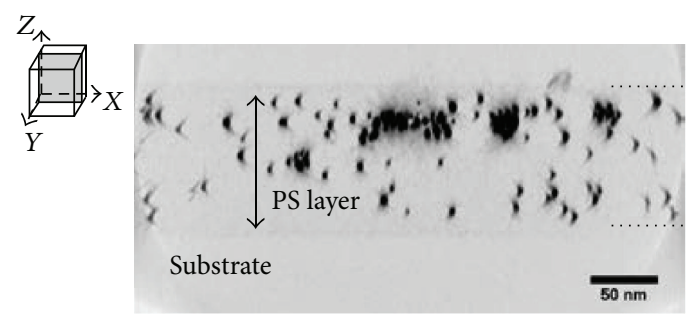

(d)

FIgURE 5: 3D analysis by electron tomography of PS@AuNPs 200 ppm: (a) PS@Au-Th and (b) PS@Au-PS bright field TEM images at $0{ }^{\circ}$ tilt from the tilt series; (c) PS@Au-Th and (d) PS@Au-PS representation of transversal sum of cross-sectional slices along one direction in the plane of 3D reconstructed volume showing different location of AuNPs within the PS layer. The upper and lower interfaces of the hybrid layer are highlighted by dotted lines. The substrate/film interface is on the bottom side of the cross-sectional views as indicated.

400 ppm concentration are presented in Figure 6. Once again, bright field TEM is unable to identify the precise location of individual or aggregated AuNPs within the bulk of the hybrid layer as seen in Figures 6(a) and 6(b). As for the previous samples, TEM tomography allows determining the precise 3D location of individual and agglomerates of AuNPs (unconnected with metallic bound) in the bulk of the sample. As seen in Figures 6(c) and 6(d), the same trends are observed as for the $200 \mathrm{ppm}$ samples: (i) a vertical phase separation with a homogeneous layer of AuNPs localized at the bottom of the PS film and the 2D agglomerates on the top of the film for the -Th ligand, (ii) individual, and agglomerates AuNPs homogeneously distributed in the bulk of the PS matrix for the -PS ligand. A slight modification can be observed in the case of PS@Au-PS for which the 3D agglomerates seem to be localized closer to the center of the PS bulk film.

A statistical analysis of the AuNPs distribution in the reconstructed volumes yields quantitative information on the densities of the NPs along the $z$-direction. The results of this analysis show that for the -Th ligand the density of individual AuNPs is about 1750 particles $/ \mu \mathrm{m}^{3}$ for $200 \mathrm{ppm}$ concentration and 4200 particles $/ \mu \mathrm{m}^{3}$ for $400 \mathrm{ppm}$ concentration, respectively, while in the case of -PS ligand the density is about 4000 particles $/ \mu \mathrm{m}^{3}$ for $200 \mathrm{ppm}$ and 7200 particles $/ \mu \mathrm{m}^{3}$ for $400 \mathrm{ppm}$ concentration, respectively.

\section{Conclusions}

This study has demonstrated (i) a versatile method to exchange the surface ligands on AuNPs by a phase transfer method to prepare stable colloidal inks with polystyrene in toluene and (ii) the impact of different surface ligands on the dispersion of AuNPs in the bulk of a polystyrene matrix in spin-coated thin films.

The method of mNPs modification with thiol-terminated polystyrene is versatile and offers flexibility regarding the NPs type (both size and material) as well as NPs surface ligand. Most importantly, the presented procedure does not require purification steps and the mNPs modified via the phase transfer process can be directly used to prepare stable inks with polystyrene in toluene as demonstrated by UV-Vis spectroscopy.

Electron tomography was used to investigate the precise dispersion of AuNPs in the bulk of a PS matrix in spin-coated thin films prepared from PS@AuNPs inks with different concentrations of AuNPs and two different types of ligands. Electron tomography shows that the use of -Th and -PS ligands leads to two distinct 3D distributions of functionalized AuNPs in the bulk of a polystyrene film (Figure 7).

For -Th ligands, a vertical phase separation between AuNPs and the PS matrix leads to a preferential location of AuNPs at the substrate/hybrid interface. Moreover, larger 


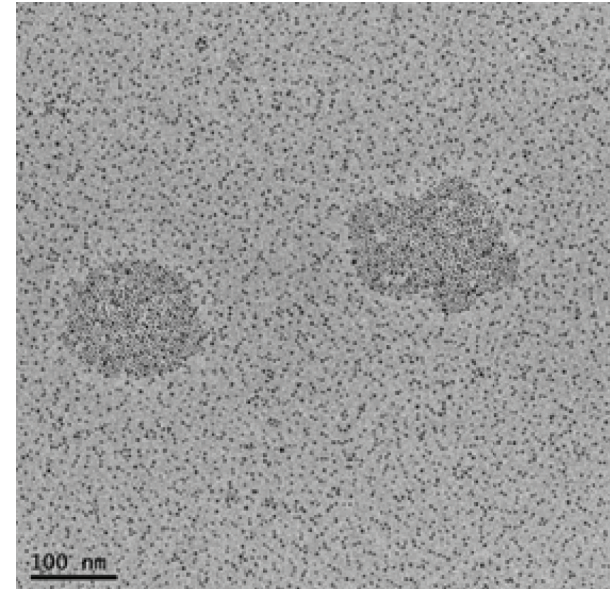

(a)

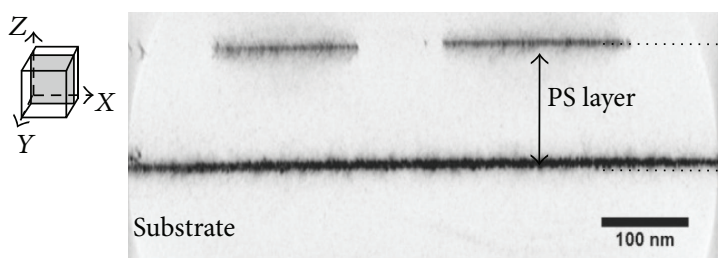

(c)

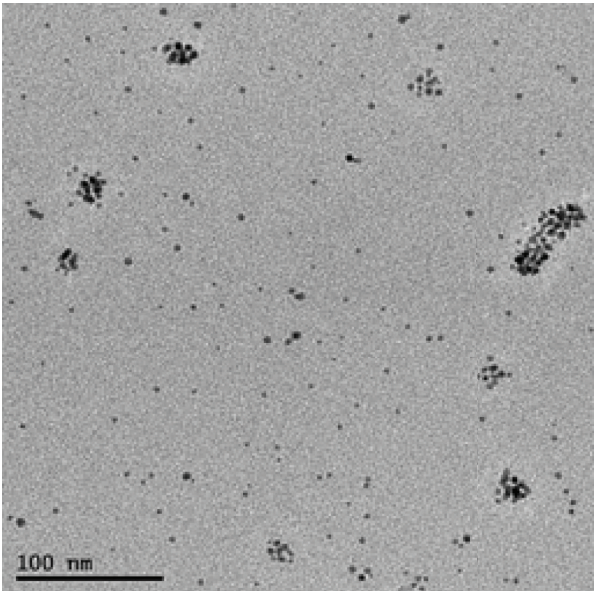

(b)



(d)

FIGURE 6: 3D analysis by electron tomography of PS@AuNPs 400 ppm: (a) PS@Au-Th and (b) PS@Au-PS bright field TEM image at $0^{\circ}$ tilt from the tilt series; (c) PS@Au-Th and (d) PS@Au-PS representation of transversal sum of cross-sectional slices along one direction in the plane of the 3D reconstructed volume showing different locations of AuNPs within the PS layer. The upper and lower interfaces of the hybrid layer are highlighted by dotted lines. The substrate/film interface is on the bottom side of the cross-sectional views as indicated.

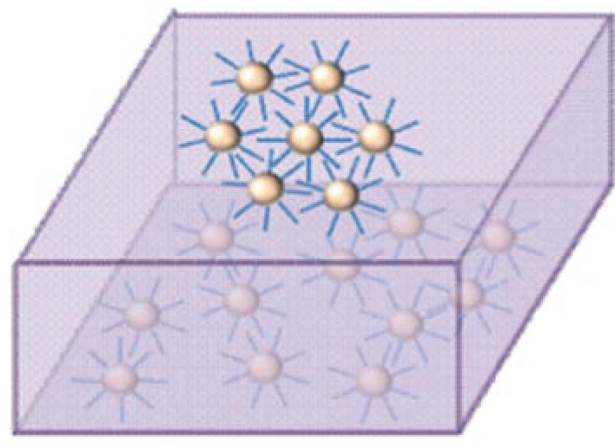

(a)

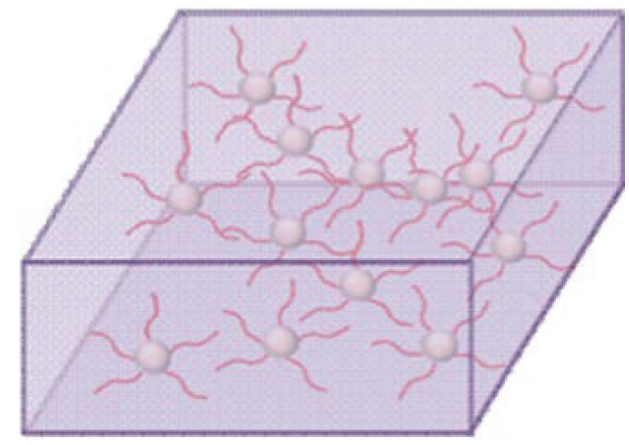

(b)

FIGURE 7: Schematic illustration of individual and aggregated AuNPs distribution within the thickness of PS layer regarding the two functionalization types: (a) -Th ligand and (b) -PS ligand. The AuNPs are schematized in yellow; the PS polymer bulk in violet; the -Th ligand in blue and -PS ligand in red.

2D agglomerates (without metallic connections between individual NPs) with regular hexagonal packing of AuNPs are observed at air/polymer interface (Figure 7(a)). These $2 \mathrm{D}$ agglomerates are possibly formed from AuNPs that were rejected at the liquid/air interface of the hybrid blends in toluene in the early stage of spin-coating. However, the presence of AuNPs agglomerates may not affect the functionality of the hybrid device as these NPs clusters will be in metallic contact with the electrode located just on the top of prepared PS@Au-Th coating. For -PS ligands, a rather homogeneous bulk dispersion of individual AuNPs is observed. Such individual dispersed AuNPs coexist with 3D agglomerates (Figure 7(b)), which are formed upon clustering of AuNPs in the bulk of the hybrid films. Overall, this study underlines the essential role played by the chemical nature of surface ligands on mNPs on their homogeneous distribution in a polymer matrix, which is a general result of interest for numerous applications based on such hybrid thin films. 


\section{Competing Interests}

The authors declare that there is no conflict of interests regarding the publication of this paper.

\section{Acknowledgments}

This work was supported by FP7-NMP-2010-SMALL-4 Program ("Hybrid Organic/Inorganic Memory Elements for Integration of Electronic and Photonic Circuitry," HYMEC), Project no. 263073. Eric Gonthier is acknowledged for technical support in the preparation of hybrid thin films. Scientific work was supported by the Polish Ministry of Science and Higher Education Funds for Science in 2011-2014 allocated for the cofunded international project.

\section{References}

[1] E. C. Wang and A. Z. Wang, "Nanoparticles and their applications in cell and molecular biology," Integrative Biology, vol. 6, no. 1, pp. 9-26, 2014.

[2] S. Karve, M. E. Werner, R. Sukumar et al., "Revival of the abandoned therapeutic wortmannin by nanoparticle drug delivery," Proceedings of the National Academy of Sciences of the United States of America, vol. 109, no. 21, pp. 8230-8235, 2012.

[3] K. Saha, S. S. Agasti, C. Kim, X. Li, and V. M. Rotello, "Gold nanoparticles in chemical and biological sensing," Chemical Reviews, vol. 112, no. 5, pp. 2739-2779, 2012.

[4] J.-M. Nam, C. S. Thaxton, and C. A. Mirkin, "Nanoparticlebased bio-bar codes for the ultrasensitive detection of proteins," Science, vol. 301, no. 5641, pp. 1884-1886, 2003.

[5] H. D. Hill and C. A. Mirkin, "The bio-barcode assay for the detection of protein and nucleic acid targets using DTTinduced ligand exchange," Nature Protocols, vol. 1, no. 1, pp. 324336, 2006.

[6] S. Schauermann, N. Nilius, S. Shaikhutdinov, and H.-J. Freund, "Nanoparticles for heterogeneous catalysis: new mechanistic insights," Accounts of Chemical Research, vol. 46, no. 8, pp. 16731681, 2013.

[7] E. Gross, J. H.-C. Liu, F. D. Toste, and G. A. Somorjai, "Control of selectivity in heterogeneous catalysis by tuning nanoparticle properties and reactor residence time," Nature Chemistry, vol. 4, no. 11, pp. 947-952, 2012.

[8] I. Piwoński and K. Soliwoda, "The effect of ceramic nanoparticles on tribological properties of alumina sol-gel thin coatings," Ceramics International, vol. 36, no. 1, pp. 47-54, 2010.

[9] I. Piwoński, K. Soliwoda, A. Kisielewska, R. Stanecka-Badura, and K. Kądzioła, "The effect of the surface nanostructure and composition on the antiwear properties of zirconia-titania coatings," Ceramics International, vol. 39, no. 2, pp. 1111-1123, 2013.

[10] G. Casula, P. Cosseddu, Y. Busby et al., "Air-stable, non-volatile resistive memory based on hybrid organic/inorganic nanocomposites," Organic Electronics: Physics, Materials, Applications, vol. 18, pp. 17-23, 2015.

[11] L. D. Bozano, B. W. Kean, M. Beinhoff, K. R. Carter, P. M. Rice, and J. C. Scott, "Organic materials and thin-film structures for cross-point memory cells based on trapping in metallic nanoparticles," Advanced Functional Materials, vol. 15, no. 12, pp. 1933-1939, 2005.
[12] J. C. Scott and L. D. Bozano, "Nonvolatile memory elements based on organic materials," Advanced Materials, vol. 19, no. 11, pp. 1452-1463, 2007.

[13] Y. N. C. Chan, G. S. W. Craig, R. R. Schrock, and R. E. Cohen, "Synthesis of palladium and platinum nanoclusters within microphase-separated diblock copolymers," Chemistry of Materials, vol. 4, no. 4, pp. 885-894, 1992.

[14] R. Saito, S.-I. Okamura, and K. Ishizu, "Introduction of colloidal silver into a poly(2-vinyl pyridine) microdomain of microphase separated poly(styrene-b-2-vinyl pyridine) film," Polymer, vol. 33, no. 5, pp. 1099-1101, 1992.

[15] B. Hamdoun, D. Ausserré, S. Joly, Y. Gallot, V. Cabuil, and C. Clinard, "New nanocomposite materials," Journal de Physique II, vol. 6, no. 4, pp. 493-501, 1996.

[16] V. Lauter-Pasyuk, H. J. Lauter, D. Ausserre et al., "Effect of nanoparticle size on the internal structure of copolymernanoparticles composite thin films studied by neutron reflection," Physica B: Condensed Matter, vol. 241-243, pp. 1092-1094, 1997.

[17] B. L. Frankamp, A. K. Boal, and V. M. Rotello, "Controlled interparticle spacing through self-assembly of Au nanoparticles and poly(amidoamine) dendrimers," Journal of the American Chemical Society, vol. 124, no. 51, pp. 15146-15147, 2002.

[18] M. Higuchi, K. Ushiba, and M. Kawaguchi, "Structural control of peptide-coated gold nanoparticle assemblies by the conformational transition of surface peptides," Journal of Colloid and Interface Science, vol. 308, no. 2, pp. 356-363, 2007.

[19] C.-A. Fustin, C. Colard, M. Filali et al., "Tuning the hydrophilicity of gold nanoparticles templated in star block copolymers," Langmuir, vol. 22, no. 15, pp. 6690-6695, 2006.

[20] D. Li, Q. He, and J. Li, "Smart core/shell nanocomposites: intelligent polymers modified gold nanoparticles," Advances in Colloid and Interface Science, vol. 149, no. 1-2, pp. 28-38, 2009.

[21] J. Raula, J. Shan, M. Nuopponen et al., "Synthesis of gold nanoparticles grafted with a thermoresponsive polymer by surface-induced reversible-addition-fragmentation chaintransfer polymerization," Langmuir, vol. 19, no. 8, pp. 3499$3504,2003$.

[22] D. Li, Q. He, Y. Cui, K. Wang, X. Zhang, and J. Li, “Thermosensitive copolymer networks modify gold nanoparticles for nanocomposite entrapment," Chemistry - A European Journal, vol. 13, no. 8, pp. 2224-2229, 2007.

[23] M. K. Corbierre, N. S. Cameron, and R. B. Lennox, "Polymerstabilized gold nanoparticles with high grafting densities," Langmuir, vol. 20, no. 7, pp. 2867-2873, 2004.

[24] A. B. Lowe, B. S. Sumerlin, M. S. Donovan, and C. L. McCormick, "Facile preparation of transition metal nanoparticles stabilized by well-defined (co)polymers synthesized via aqueous reversible addition-fragmentation chain transfer polymerization," Journal of the American Chemical Society, vol. 124, no. 39, pp. 11562-11563, 2002.

[25] S. Mössmer, J. P. Spatz, M. Möller, T. Aberle, J. Schmidt, and W. Burchard, "Solution behavior of poly(styrene)-blockpoly(2-vinylpyridine) micelles containing gold nanoparticles," Macromolecules, vol. 33, no. 13, pp. 4791-4798, 2000.

[26] J. Q. Lu and S. S. Yi, "Uniformly sized gold nanoparticles derived from PS-b-P2VP block copolymer templates for the controllable synthesis of Si nanowires," Langmuir, vol. 22, no. 9, pp. 3951-3954, 2006.

[27] D. Li, Ch. Li, A. Wang, Q. He, and J. Li, "Hierarchical gold/ copolymer nanostructures as hydrophobic nanotanks for drug 
encapsulation," Journal of Materials Chemistry, vol. 20, no. 36, pp. 7782-7787, 2010.

[28] G. Yang, W.-S. Chang, and D. T. Hallinan Jr., "A convenient phase transfer protocol to functionalize gold nanoparticles with short alkylamine ligands," Journal of Colloid and Interface Science, vol. 460, pp. 164-172, 2015.

[29] B. Tkacz-Szczesna, K. Soliwoda, M. Rosowski, E. Tomaszewska, G. Celichowski, and J. Grobelny, "Modification of gold and silver nanoparticles with $n$-dialkyldithiophosphates," Colloids and Surfaces A: Physicochemical and Engineering Aspects, vol. 468, pp. 219-225, 2015.

[30] K. Soliwoda, E. Tomaszewska, B. Tkacz-Szczesna, M. Rosowski, G. Celichowski, and J. Grobelny, "The influence of the chain length and the functional group steric accessibility of thiols on the phase transfer efficiency of gold nanoparticles from water to toluene," Polish Journal of Chemical Technology, vol. 16, no. 1, pp. 86-91, 2014.

[31] K. Soliwoda, E. Tomaszewska, B. Tkacz-Szczesna et al., "Effect of the alkyl chain length of secondary amines on the phase transfer of gold nanoparticles from water to toluene," Langmuir, vol. 30, no. 23, pp. 6684-6693, 2014.

[32] N. G. Bastús, F. Merkoçi, J. Piella, and V. Puntes, "Synthesis of highly monodisperse citrate-stabilized silver nanoparticles of up to $200 \mathrm{~nm}$ : kinetic control and catalytic properties," Chemistry of Materials, vol. 26, no. 9, pp. 2836-2846, 2014.

[33] M. Brust, M. Walker, D. Bethell, D. J. Schiffrin, and R. Whyman, "Synthesis of thiol-derivatised gold nanoparticles in a twophase liquid-liquid system," Journal of the Chemical Society, Chemical Communications, no. 7, pp. 801-802, 1994.

[34] S. R. K. Perala and S. Kumar, "On the mechanism of metal nanoparticle synthesis in the Brust-Schiffrin method," Langmuir, vol. 29, no. 31, pp. 9863-9873, 2013.

[35] X. M. Lin, C. M. Sorensen, and K. J. Klabunde, "Digestive ripening, nanophase segregation and superlattice formation in gold nanocrystal colloids," Journal of Nanoparticle Research, vol. 2, no. 2, pp. 157-164, 2000.

[36] R. Shankar, B. B. Wu, and T. P. Bigioni, "Wet chemical synthesis of monodisperse colloidal silver nanocrystals using digestive ripening," Journal of Physical Chemistry C, vol. 114, no. 38, pp. 15916-15923, 2010.

[37] M. K. Corbierre, N. S. Cameron, M. Sutton et al., "Polymerstabilized gold nanoparticles and their incorporation into polymer matrices," Journal of the American Chemical Society, vol. 123, no. 42, pp. 10411-10412, 2001.

[38] M. K. Corbierre, N. S. Cameron, M. Sutton, K. Laaziri, and R. B. Lennox, "Gold nanoparticle/polymer nanocomposites: dispersion of nanoparticles as a function of capping agent molecular weight and grafting density," Langmuir, vol. 21, no. 13, pp. 6063-6072, 2005.

[39] J. M. Stouffer and T. J. McCarthy, "Polymer monolayers prepared by the spontaneous adsorption of sulfur-functionalized polystyrene on gold surfaces," Macromolecules, vol. 21, no. 5, pp. 1204-1208, 1988.

[40] H. Dohi, H. Kimura, M. Kotani et al., "Three-dimensional imaging in polymer science: its application to block copolymer morphologies and rubber composites," Polymer Journal, vol. 39, no. 8, pp. 749-758, 2007.

[41] H. Jinnai, R. J. Spontak, and T. Nishi, "Transmission electron microtomography and polymer nanostructures," Macromolecules, vol. 43, no. 4, pp. 1675-1688, 2010.

[42] F. Dalmas, N. Genevaz, M. Roth, J. Jestin, and E. Leroy, "3D dispersion of spherical silica nanoparticles in polymer nanocomposites: a quantitative study by electron tomography," Macromolecules, vol. 47, no. 6, pp. 2044-2051, 2014.

[43] S. Akasaka, H. Mori, T. Osaka, V. H. Mareau, and H. Hasegawa, "Controlled introduction of metal nanoparticles into a microdom structure," Macromolecules, vol. 42, no. 4, pp. 1194$1202,2009$.

[44] M. K. Mayeda, W.-F. Kuan, W.-S. Young, J. A. Lauterbach, and T. H. Epps III, "Controlling particle location with mixed surface functionalities in block copolymer thin films," Chemistry of Materials, vol. 24, no. 14, pp. 2627-2634, 2012.

[45] M. Fu, A. Wang, X. Zhang, L. Dai, and J. Li, "Direct observation of the distribution of gelatin in calcium carbonate crystals by super-resolution fluorescence microscopy," Angewandte Chemie-International Edition, vol. 55, no. 3, pp. 908-911, 2016.

[46] A. I. Frenkel, S. Nemzer, I. Pister et al., "Size-controlled synthesis and characterization of thiol-stabilized gold nanoparticles," Journal of Chemical Physics, vol. 123, no. 18, Article ID 184701, 2005.

[47] J. R. Kremer, D. N. Mastronarde, and J. R. McIntosh, "Computer visualization of three-dimensional image data using IMOD," Journal of Structural Biology, vol. 116, no. 1, pp. 71-76, 1996.

[48] C. MessaoudiI, T. Boudier, C. O. S. Sorzano, and S. Marco, "TomoJ: tomography software for three-dimensional reconstruction in transmission electron microscopy," BMC Bioinformatics, vol. 8, article 288, 2007.

[49] T. Sakura and Y. Nagasaki, "Preparation of gold colloid using pyrrole-2-carboxylic acid and characterization of its particle growth," Colloid and Polymer Science, vol. 285, no. 12, pp. 14071410, 2007. 

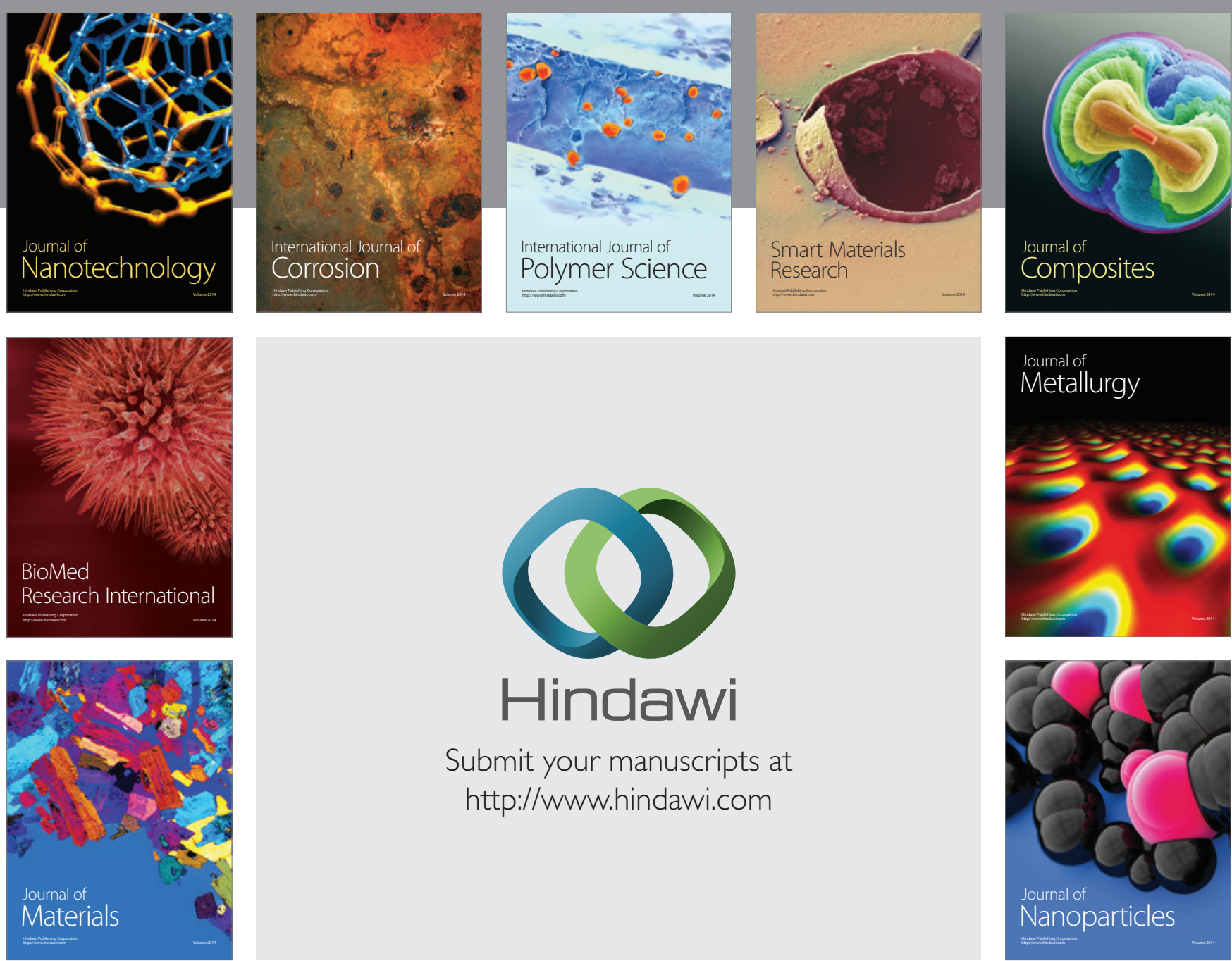

\section{Hindawi}

Submit your manuscripts at

http://www.hindawi.com

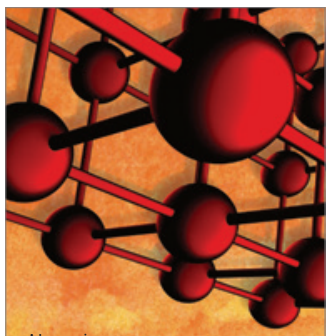

Materials Science and Engineering
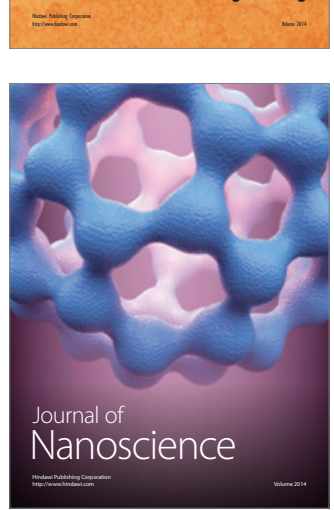

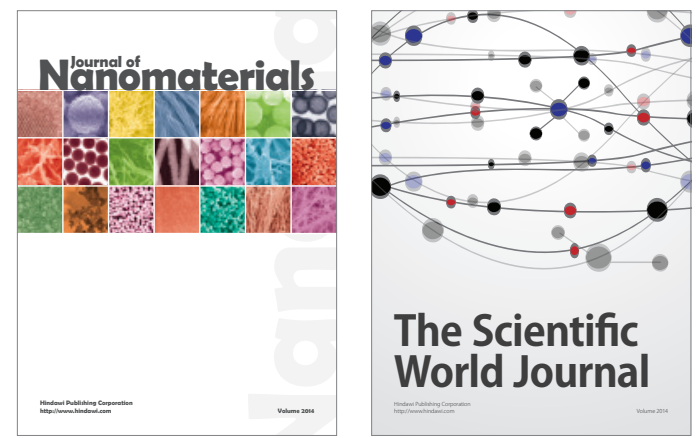

The Scientific World Journal
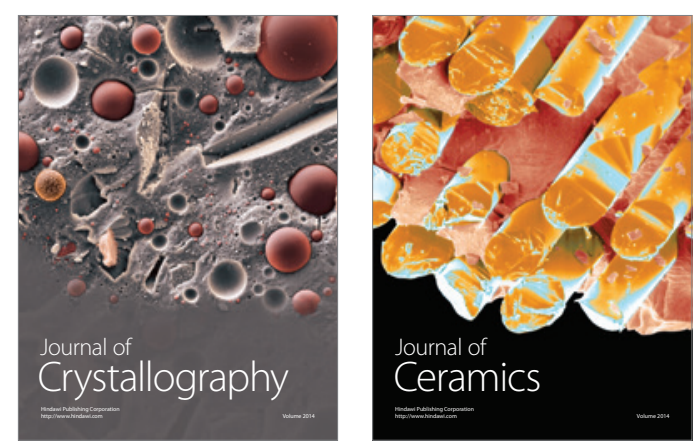
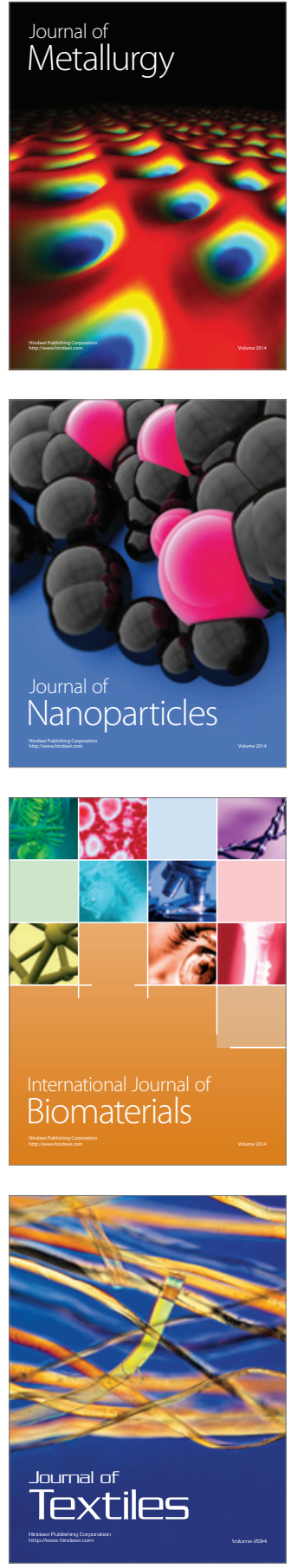